\title{
INVOLUTION AND THE HAAGERUP TENSOR PRODUCT
}

\author{
AJAY KUMAR \\ Department of Mathematics, Rajdhani College (University of Delhi), \\ Raja Garden, New Delhi 110015, India (ajaykr@bol.net.in)
}

(Received 13 July 1999)

\begin{abstract}
We show that the involution $\theta(a \otimes b)=a^{*} \otimes b^{*}$ on the Haagerup tensor product $A \otimes_{\mathrm{H}} B$ of $C^{*}$-algebras $A$ and $B$ is an isometry if and only if $A$ and $B$ are commutative. The involutive Banach algebra $A \otimes_{\mathrm{H}} A$ arising from the involution $a \otimes b \rightarrow b^{*} \otimes a^{*}$ is also studied.

Keywords: $C^{*}$-algebras; Haagerup tensor product; second dual; closed ideals
\end{abstract}

AMS 2000 Mathematics subject classification: Primary 46L05; 46M05

\section{Introduction}

The Haagerup norm of an element $u$ in the algebraic tensor product $A \otimes B$ of two $C^{*}$ algebras $A$ and $B$ is defined by

$$
\|u\|_{\mathrm{H}}=\inf \left\|\sum_{j=1}^{n} a_{j} a_{j}^{*}\right\|^{1 / 2}\left\|\sum_{j=1}^{n} b_{j}^{*} b_{j}\right\|^{1 / 2}=\inf \left\|\left(a_{1}, a_{2}, \ldots, a_{n}\right)\right\|\left\|\left(b_{1}, b_{2}, \ldots, b_{n}\right)^{\prime}\right\|,
$$

where these infima are taken over all representations of $u=\sum_{j=1}^{n} a_{j} \otimes b_{j}, a_{j} \in A, b_{j} \in B$, and $\left(b_{1}, b_{2}, \ldots, b_{n}\right)^{\prime}$ is the transpose of the row operator. The Haagerup tensor product $A \otimes_{\mathrm{H}} B$ is the Banach space obtained by completing the algebraic tensor product $A \otimes B$ in the Haagerup norm. A direct calculation with the definition and Cauchy-Schwarz inequality shows that $A \otimes_{\mathrm{H}} B$ is a Banach algebra with the natural multiplication $(a \otimes$ $b)(x \otimes y)=a x \otimes b y, a, x \in A$ and $b, y \in B[\mathbf{3}]$. The Haagerup tensor product $A \otimes_{\mathrm{H}} B$ is a $C^{*}$-algebra if and only if $A$ or $B$ equals $\mathbb{C}$ [4]. This tensor product plays an important role in the theory of operator spaces $[\mathbf{4}-\mathbf{6}, \mathbf{8}, \mathbf{9}]$ and is an injective tensor product $[\mathbf{1 3}]$. The ideal structure of this Banach algebra has been studied in [1] and [2].

First we show that a natural involution $\theta: A \otimes B \rightarrow A \otimes B$ given by $\theta(a \otimes b)=a^{*} \otimes b^{*}$ lifts to a continuous map $\theta_{\mathrm{H}}$ on $A \otimes_{\mathrm{H}} B$ if and only if either $A$ or $B$ is finite dimensional, or $A$ and $B$ are infinite dimensional and subhomogeneous. Recall that a $C^{*}$-algebra is subhomogeneous if for some $k \in N$, every irreducible representation is on a Hilbert space of dimension not greater than $k$. Furthermore, it has been shown that $\theta_{\mathrm{H}}$ is an isometry if and only if $A$ and $B$ are commutative. It follows from the definition of the Haagerup norm that the Haagerup tensor product $A \otimes_{\mathrm{H}} A$ is an involutive Banach algebra with 
isometric involution given by $a \otimes b \rightarrow b^{*} \otimes a^{*}$. For a unital $C^{*}$-algebra $A$, we show that if $A \otimes_{\mathrm{H}} A$ has a faithful ${ }^{*}$-representation on a Hilbert space, then $A$ is commutative. As a corollary it follows that $A \otimes_{\mathrm{H}} A$ is ${ }^{*}$-semi simple (Hermitian) if and only if $A$ is commutative. Finally, the closed ${ }^{*}$-ideals of $A \otimes_{\mathrm{H}} A$ are studied.

\section{Results}

For a Banach space $X, X^{*}$ denotes the dual of $X$. Let $M_{n}$ be the $C^{*}$-algebra of $n \times n$ complex matrices acting on the $n$-dimensional complex Hilbert space $\mathbb{C}^{n}$. For a complex Hilbert space $H$, let $B(H)$ be the algebra of bounded operators on $H$ and $K(H)$ the ideal of compact operators. The following lemma is proved in [12] using the Cauchy-Schwarz inequality and the action of $M_{n} \otimes_{\mathrm{H}} M_{n}$ on $M_{n}$ as completely bounded operators.

Lemma 2.1. For $n \in N$, if $e_{i j}$ for $1 \leqslant i, j \leqslant n$ are the matrix units in $M_{n}$ and $l_{n}^{\infty}$ is the diagonal algebra in $M_{n}$, then

$$
\left\|\sum_{j=1}^{n} e_{1 j} \otimes e_{j j}\right\|_{\mathrm{H}}=n^{1 / 2} \quad \text { and } \quad\left\|\sum_{j=1}^{n} e_{j 1} \otimes e_{j j}\right\|_{\mathrm{H}}=1
$$

in $M_{n} \otimes_{\mathrm{H}} l_{n}^{\infty}$. Also in $l_{n}^{\infty} \otimes_{\mathrm{H}} M_{n}$

$$
\left\|\sum_{j=1}^{n} e_{j j} \otimes e_{j 1}\right\|_{\mathrm{H}}=n^{1 / 2} \quad \text { and } \quad\left\|\sum_{j=1}^{n} e_{j j} \otimes e_{1 j}\right\|_{\mathrm{H}}=1 .
$$

Theorem 2.2. Let $A$ and $B$ be $C^{*}$-algebras and $\theta$ is the map on $A \otimes B$ given by $\theta(a \otimes b)=a^{*} \otimes b^{*}$. Then the following are equivalent.

(i) The Haagerup norm $\|\cdot\|_{\mathrm{H}}$ is equivalent to the Banach space projective norm $\|\cdot\|_{\gamma}$.

(ii) $\theta$ lifts to a continuous map $\theta_{\mathrm{H}}$ on $A \otimes_{\mathrm{H}} B$.

(iii) Either $A$ or $B$ is finite dimensional or $A$ and $B$ are infinite dimensional and subhomogeneous.

Proof. The equivalence of (i) and (iii) is shown in [12]. It is trivial that (i) implies (ii). We now show that (ii) implies (iii). Suppose that $\theta$ lifts to a continuous map $\theta_{\mathrm{H}}$ on $A \otimes_{\mathrm{H}} B$ and $A$ and $B$ are infinite dimensional. Then $\theta_{\mathrm{H}}$ is a continuous map on $\left(A \otimes_{\mathrm{H}} B\right)^{* *}$ which contains $A^{* *} \otimes_{\mathrm{H}} B^{* *}[\mathbf{5}, \mathbf{1 0}]$. For $u \in\left(A \otimes_{\mathrm{H}} B\right)^{* *}$ and $\phi \in\left(A \otimes_{\mathrm{H}} B\right)^{*}$, $\left(\theta_{\mathrm{H}} u\right)(\phi)=u\left(\phi \circ \theta_{\mathrm{H}}\right)$.

The dual space of the Haagerup tensor product of two $C^{*}$-algebras is the space of completely bounded bilinear forms on the algebras [9]. So, by [9], for $\phi \in\left(A \otimes_{\mathrm{H}} B\right)^{*}$ there exist Hilbert spaces $H$ and $K$, representations $\pi_{1}: A \rightarrow B(H)$ and $\pi_{2}: B \rightarrow B(K)$, vectors $\xi \in K$ and $\eta \in H$, and a bounded linear operator $T: K \rightarrow H$ such that

$$
\phi(x \otimes y)=\left\langle\pi_{1}(x) T \pi_{2}(y) \xi, \eta\right\rangle
$$


for all $x \in A, y \in B$. Assuming that the representations $\pi_{1}$ and $\pi_{2}$ of $A$ and $B$ are faithful, we can identify $A$ with $\pi_{1}(A)$ and $B$ with $\pi_{2}(B)$. The above expression can be rewritten as

$$
\phi(x \otimes y)=\langle x T y \xi, \eta\rangle
$$

for all $x \in A \subseteq B(H), y \in B \subseteq B(K)$. For $v \in A^{* *}, \omega \in B^{* *}$, the element $v \otimes \omega$ of $A^{* *} \otimes_{\mathrm{H}} B^{* *}$ can be viewed as an element of $\left(A \otimes_{\mathrm{H}} B\right)^{* *}$ by

$$
v \otimes \omega(\phi)=\langle v T \omega \xi, \eta\rangle .
$$

This inclusion is an isometry $[\mathbf{5}, \mathbf{1 0}]$. Thus $\theta_{\mathrm{H}}^{* *}(v \otimes \omega)=v^{*} \otimes \omega^{*}$.

If for some $\eta \in N$, the von Neumann algebras $A^{* *}$ or $B^{* *}$ (say $A^{* *}$ ) contain an isomorphic copy of $M_{n}$, then, by Lemma 2.1,

$$
n^{1 / 2}=\left\|\sum_{j=1}^{n} e_{1 j} \otimes e_{j j}\right\|_{\mathrm{H}}=\left\|\theta_{\mathrm{H}}^{* *}\left(\sum_{j=1}^{n} e_{j 1} \otimes e_{j j}\right)\right\|_{\mathrm{H}} \leqslant\left\|\theta_{\mathrm{H}}^{* *}\right\|\left\|\sum_{j=1}^{n} e_{j 1} \otimes e_{j j}\right\|_{\mathrm{H}}=\left\|\theta_{\mathrm{H}}^{* *}\right\|_{\mathrm{H}},
$$

by the injectivity of the Haagerup norm [13]. It follows that $A^{* *}$ and $B^{* *}$ cannot contain a type $I_{n}$ factor for $n>\left\|\theta_{\mathrm{H}}\right\|^{2}$. So, $A^{* *}$ and $B^{* *}$ are of the form $\oplus N_{j}, j \leqslant\left\|\theta_{\mathrm{H}}\right\|^{2}$, where $N_{j}$ is a von Neumann algebra of type $I_{j}$. If $\pi$ is an irreducible representation of $A$ on a Hilbert space $H$, there is a normal representation $\pi$ of $A^{* *}$ on $H$ such that $\pi\left(A^{* *}\right)=\overline{\pi(A)}$ (weak closure $)=B(H)$. Hence, $\operatorname{dim} H \leqslant\left\|\theta_{\mathrm{H}}\right\|^{2}$. Similarly, $B$ is also subhomogeneous.

Theorem 2.3. Let $A$ and $B$ be infinite-dimensional $C^{*}$-algebras and $\theta(a \otimes b)=a^{*} \otimes b^{*}$. If $\theta$ lifts to a continuous map $\theta_{\mathrm{H}}$ on $A \otimes_{\mathrm{H}} B$, then $\theta_{\mathrm{H}}$ is an isometry if and only if $A$ and $B$ are commutative.

Proof. If $A$ and $B$ are commutative, by the definition of the Haagerup norm, $\theta_{\mathrm{H}}$ is an isometry. Conversely, if $\theta_{\mathrm{H}}$ is an isometry on $A \otimes_{\mathrm{H}} B$, then $\theta_{\mathrm{H}}$ lifts to an isometry $\theta_{\mathrm{H}}^{* *}$ on $\left(A \otimes_{\mathrm{H}} B\right)^{* *}$. As in Theorem 2.2, $\theta_{\mathrm{H}}^{* *}(v \otimes \omega)=v^{*} \otimes \omega^{*}$ for all $v \in A^{* *}$ and $\omega \in B^{* *}$. If at least one of the von Neumann algebras $A^{* *}$ or $B^{* *}$ is not commutative, say $A^{* *}$, then by the decomposition of a von Neumann algebra into type $\mathrm{I}, \mathrm{II}_{1}, \mathrm{II}_{\infty}$, III, it follows that $A^{* *} \supset M_{n}$ for some $n>1[\mathbf{1 1}]$. Lemma 2.1 and the injectivity of Haagerup norm [13] now show that $\theta_{\mathrm{H}}^{* *}$ is not an isometry. Hence $A^{* *}$ and $B^{* *}$ are commutative, and in particular so are $A$ and $B$.

Let $A$ be a $C^{*}$-algebra. By the definition of Haagerup norm, $A \otimes_{\mathrm{H}} A$ is a Banach ${ }^{*}$ algebra with isometric involution given by $a \otimes b \rightarrow b^{*} \otimes a^{*}, a, b \in A$. For a Hilbert space $H, \pi: A \otimes_{\mathrm{H}} A \rightarrow B(H)$ will be called a ${ }^{*}$-representation if $\pi$ is a bounded algebraic homomorphism satisfying $\pi\left(b^{*} \otimes a^{*}\right)=(\pi(a \otimes b))^{*}$ for all $a, b \in A$. If, in addition, $\pi\left(A \otimes_{\mathrm{H}} A\right)$ is $\sigma$-weakly dense in $B(H)$, then $\pi$ is said to be irreducible.

Theorem 2.4. Let $A$ be a unital $C^{*}$-algebra. If $A \otimes_{\mathrm{H}} A$ has a faithful ${ }^{*}$-representation, then $A$ is commutative. 
Proof. Let $\pi$ be a faithful ${ }^{*}$-representation of $A \otimes_{\mathrm{H}} A$ on a Hilbert space $H$. Putting $\pi_{1}(a)=\pi(a \otimes 1)$ and $\pi_{2}(a)=\pi(1 \otimes a), a \in A$, it is easy to verify that $\pi_{1}$ and $\pi_{2}$ are bounded monomorphisms from $A$ into $B(H)$ satisfying $\pi(a \otimes b)=\pi_{1}(a) \pi_{2}(b)=$ $\pi_{2}(b) \pi_{1}(a)$ for all $a, b \in A$ and $\pi_{1}\left(a^{*}\right)=\pi_{2}(a)^{*}, a \in A$. If $h$ is a self-adjoint element of $A$, then $\| \exp$ ith $\|=1$ for all $t \in \mathbb{R}$. The ${ }^{*}$-homomorphism $\pi$ from the Banach ${ }^{*}$-algebra $A \otimes_{\mathrm{H}} A$ to $B(H)$ is norm reducing [14, Proposition 1.5.2]. Thus

$$
\left\|\exp \mathrm{i} t \pi_{1}(h)\right\|=\|\pi(\exp \mathrm{i} t(h \otimes 1))\| \leqslant\|\exp \mathrm{i} t(h \otimes 1)\|_{\mathrm{H}}=\|\exp \mathrm{i} t h\|=1,
$$

for all $t \in \mathbb{R}$. Hence, $\left\|\exp \mathrm{i} t \pi_{1}(h)\right\|=1$ for all $t \in \mathbb{R}$. So $\pi_{1}(h)$ is a self-adjoint element of $B(H)$. Let $a=h+\mathrm{i} k$, where $h$ and $k$ are self-adjoint elements of $A$. Now

$$
\pi_{1}\left(a^{*}\right)=\pi_{1}(h-i k)=\pi_{1}(h)-i \pi_{1}(k)=\left(\pi_{1}(h)+i \pi_{1}(k)\right)^{*}=\left(\pi_{1}(a)\right)^{*} .
$$

This implies that $\pi_{1}\left(a^{*}\right)=\pi_{1}(a)^{*}=\pi_{2}(a)^{*}$ for all $a \in A$ and, thus, $\pi_{1}(a)=\pi_{2}(a)$ for all $a \in A$. But $\pi_{1}(a) \pi_{2}(b)=\pi_{2}(b) \pi_{1}(a)$, so $\pi(a b-b a \otimes 1)=\pi_{1}(a b-b a)=0$ for all $a, b \in A$. Since $\pi$ is faithful, we have $a b-b a=0$ for all $a, b \in A$, i.e. $A$ is commutative.

It is well known for a $C^{*}$-algebra $A, \cap\left\{\operatorname{ker} \pi: \pi\right.$ is a ${ }^{*}$-representation of $\left.A\right\}=\{0\}$, i.e. $A$ is ${ }^{*}$-semi simple. An equivalent form of the above result is the following.

Corollary 2.5. Let $A$ be a unital $C^{*}$-algebra. Then $A \otimes_{\mathrm{H}} A$ is ${ }^{*}$-semi simple if and only if $A$ is commutative.

Recall that a Banach *-algebra $A$ is said to be Hermitian if every self-adjoint element of $A$ has real spectrum [7]. Moreover, in a Hermitian Banach *-algebra $A$, the radical of $A$ equals the star radical of $A$ [7, Theorem 4.9]. Since $\operatorname{rad}\left(A \otimes_{\mathrm{H}} A\right)=(0)$ by $[\mathbf{1}$, Proposition 5.16], we have the following.

Corollary 2.6. Let $A$ be a unital $C^{*}$-algebra. Then $A \otimes_{\mathrm{H}} A$ is Hermitian if and only if $A$ is commutative.

A careful reading of the proof of Theorem 2.4 shows the following.

Proposition 2.7. Let $A$ is a unital $C^{*}$-algebra and $\pi$ a $^{*}$-representation of $A \otimes_{\mathrm{H}} A$, then there is a ${ }^{*}$-representation $\pi_{0}$ of $A$ satisfying $\pi(a \otimes b)=\pi_{0}(a b)$ and $\pi_{0}(A)$ is abelian.

Suppose that $A$ is a $C^{*}$-algebra having only a finite number of closed two-sided ideals. Let $K$ be a closed *-ideal of $A \otimes_{\mathrm{H}} A$. By [1, Theorem 5.3], $K=\sum_{j}\left(K_{j} \otimes_{\mathrm{H}} I_{j}\right)$, where $K_{j}, I_{j}$ are closed ideals of $A$ and, hence, ${ }^{*}$-ideals. Thus any *-ideal of $A \otimes_{\mathrm{H}} A$ is of the form

$$
\sum_{j}\left(K_{j} \otimes_{\mathrm{H}} I_{j}+I_{j} \otimes_{\mathrm{H}} K_{j}\right)
$$

In particular, the only closed proper *-ideals of $B(H) \otimes_{\mathrm{H}} B(H)$ are $B(H) \otimes_{\mathrm{H}} K(H)+$ $K(H) \otimes_{\mathrm{H}} B(H)$ and $K(H) \otimes_{\mathrm{H}} K(H)$.

Our next result characterizes the ${ }^{*}$-ideals of $A \otimes_{\mathrm{H}} A$ annihilated by a *-representation of $A \otimes_{\mathrm{H}} A$. 
Theorem 2.8. Let $A$ be a unital $C^{*}$-algebra. Then a closed two-sided *-ideal $J$ of $A \otimes_{\mathrm{H}} A$ is annihilated by a ${ }^{*}$-representation $\pi$ of $A \otimes_{\mathrm{H}} A$ if and only if there is a ${ }^{*}$ representation $\pi_{0}$ of $A$ with $\pi_{0}(A)$ abelian such that $J \subseteq J_{0} \otimes_{\mathrm{H}} A+A \otimes_{\mathrm{H}} J_{0}, J_{0}=\operatorname{ker} \pi_{0}$.

Proof. Suppose that $J \subseteq \operatorname{ker} \pi$, where $\pi$ is a ${ }^{*}$-representation of $A \otimes_{\mathrm{H}} A$ on a Hilbert space $H$. Let $\pi_{0}$ be a ${ }^{*}$-representation of $A$ as in Proposition 2.7 and $J_{0}=\operatorname{ker} \pi_{0}$. Clearly, ker $\pi \supseteq J_{0} \otimes_{\mathrm{H}} A+A \otimes_{\mathrm{H}} J_{0}$ and $A / J_{0} \otimes_{\mathrm{H}} A / J_{0}$ is commutative. Let $q: A \otimes_{\mathrm{H}} A \rightarrow$ $A / J_{0} \otimes_{\mathrm{H}} A / J_{0}$ be the quotient map with kernel $J_{0} \otimes_{\mathrm{H}} A+A \otimes_{\mathrm{H}} J_{0}$. The representation $\pi$ induces a faithful representation $\pi_{0}$ of $A / J_{0} \otimes_{\mathrm{H}} A / J_{0}$ on $H$. Moreover, the following diagram commutes.

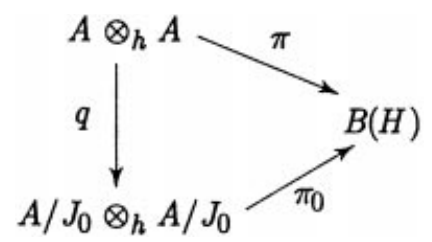

So $\pi(J)=0$ implies that $q(J)=0$. Thus $J \subseteq J_{0} \otimes_{\mathrm{H}} A+A \otimes_{\mathrm{H}} J_{0}$. Conversely, suppose that $J \subseteq J_{0} \otimes_{\mathrm{H}} A+A \otimes_{\mathrm{H}} J_{0}, J_{0}=\operatorname{ker} \pi_{0}, \pi_{0}$ is a ${ }^{*}$-representation of $A$ with $\pi_{0}(A)$ abelian. Defining $\pi$ by $\pi(a \otimes b)=\pi_{0}(a b)$ on $A \otimes_{\mathrm{H}} A$, it is easy to verify that $\pi$ is a *-representation of $A \otimes_{\mathrm{H}} A$ and $J \subseteq \operatorname{ker} \pi$.

Let $H$ be a separable infinite-dimensional Hilbert space, it follows from the above theorem that the ${ }^{*}$-ideal $K(H) \otimes_{\mathrm{H}} K(H)$ cannot be annihilated by a ${ }^{*}$-representation of $B(H) \otimes_{\mathrm{H}} B(H)$.

In contrast to Theorem 2.8, if the involution $a \otimes b \rightarrow b^{*} \otimes a^{*}$ is dropped, then of course for every proper closed two-sided ideal $J$ there is a bounded algebraic homomorphism $\pi: A \otimes_{\mathrm{H}} A \rightarrow B(H)$, satisfying $\pi\left(a^{*} \otimes b^{*}\right)=\pi(a \otimes b)^{*}, a, b \in A$ such that $J \subseteq \operatorname{ker} \pi$. The proof of this result is implicitly contained in [1] (see also [2]), but to be more explicit, we outline the proof.

Theorem 2.9. Let $A$ and $B$ be unital $C^{*}$-algebras. Then every proper closed two-sided ideal of $A \otimes_{\mathrm{H}} B$ is annihilated by a representation of $A \otimes_{\mathrm{H}} B$.

Proof. Let $J$ be a proper closed two-sided ideal of $A \otimes_{\mathrm{H}} B$ and $J_{\min }$ be the closure of $J$ in $A \otimes_{\min } B$, where $A \otimes_{\min } B$ is the completion of the algebraic tensor product with $\|\cdot\|_{\min }$ norm. If $J_{\min }=A \otimes_{\min } B$, then $J_{\min }$ will contain all elementary tensors, so, by [1, Theorem 4.4], $J$ will be equal to $A \otimes_{\mathrm{H}} B$. Thus, $J_{\min }$ is a proper closed two-sided ideal in the $C^{*}$-algebra $A \otimes_{\min } B$. Let $\pi$ be an irreducible representation of $A \otimes_{\min } B$ on a Hilbert space $H$ annihilating $J_{\min }$. Let $\pi_{1}(a)=\pi(a \otimes 1)$ and $\pi_{2}(b)=\pi(1 \otimes b)$, for all $a \in A$ and $b \in B$. Then $\pi_{1}$ and $\pi_{2}$ are commuting representations of $A$ and $B$, respectively. Let $M=\operatorname{ker} \pi_{1}$ and $N=\operatorname{ker} \pi_{2}$. Let $q: A \otimes_{\mathrm{H}} B \rightarrow A / M \otimes_{\mathrm{H}} B / N$ be the quotient map and let $\pi_{1} \cdot \pi_{2}: A / M \otimes_{\mathrm{H}} B / N \rightarrow B(H)$ be the faithful representation of $A / M \otimes_{\mathrm{H}} B / N$ induced by $\pi_{1}$ and $\pi_{2}$ (see [1] for details). So $\pi(J)=0$ implies that $q(J)=0$. But $A / M \otimes_{\mathrm{H}} B / N \simeq A \otimes_{\mathrm{H}} B / M \otimes_{\mathrm{H}} B+A \otimes_{\mathrm{H}} N$, thus $J \subseteq M \otimes_{\mathrm{H}} B+A \otimes_{\mathrm{H}} N$. Since $M \otimes_{\mathrm{H}} B+A \otimes_{\mathrm{H}} N$ is primitive [1, Theorem 5.13], there is a representation $\sigma$ of $A \otimes_{\mathrm{H}} B$ such that $J \subseteq \operatorname{ker} \sigma$. 
Acknowledgements. The author is grateful to Professor A. M. Sinclair for many valuable comments.

\section{References}

1. S. D. Allen, A. M. Sinclair and R. R. Smith, The ideal structure of the Haagerup tensor product of $C^{*}$-algebras, J. Reine Angew. Math. 442 (1993), 111-148.

2. R. J. Archbold, D. W. B. Somerset, E. Kaniuth And G. Schlichting, Ideal spaces of the Haagerup tensor product of $C^{*}$-algebras, Int. J. Math. 8 (1997), 1-29.

3. D. P. Blecher, Geometry of tensor product of $C^{*}$-algebras, Math. Proc. Camb. Phil. Soc. 104 (1988), 119-127.

4. D. P. BleCher AND V. I. PAUlsen, Tensor product of operator spaces, J. Funct. Analysis 99 (1991), 262-292.

5. D. P. Blecher And R. R. Smith, The dual of the Haagerup tensor product, J. Lond. Math. Soc. 45 (1992), 126-144.

6. D. P. Blecher, Z. J. Ruan And A. M. Sinclair, A characterization of operator algebras, J. Funct. Analysis 89 (1990), 188-201.

7. F. F. Bonsall and J. Duncan, Complete normed algebras (Berlin: Springer, 1973).

8. E. Christensen AND A. M. Sinclair, A survey of completely bounded operators, Bull. Lond. Math. Soc. 21 (1989), 417-448.

9. E. G. Effros And A. Kishimoto, Module maps and Hochschild-Johnson cohomology, Indiana Univ. Math. J. 36 (1987), 257-276.

10. E. G. Effros And Z. J. RUAn, Self duality for the Haagerup tensor product and Hilbert space factorization, J. Funct. Analysis. 100 (1991), 257-284.

11. R. V. Kadison And J. R. Ringrose, Fundamentals of the theory of operator algebras, vol. II (Academic, 1983).

12. A. KUMAR AND A. M. Sinclair, Equivalence of norms on operator space tensor products of $C^{*}$-algebras, Trans. Am. Math. Soc. 350 (1998) 2033-2048.

13. V. I. PAUlsen And R. R. Smith, Multilinear maps and tensor norms on operator systems, J. Funct. Analysis $\mathbf{7 3}$ (1987), 258-276.

14. M. TAKESAKI, Theory of operator algebras, vol. I (Berlin: Springer, 1979). 